\title{
Nature and outcome of psychotic illness was similar in all ethnic groups
}

Goater N, King M, Cole E, et al. Ethnicity and outcome of psychosis. Br J Psychiatry 1999 Jul;175:34-42.

QUESTION: What is the long term outcome of psychosis in different ethnic groups?

Design

Inception cohort followed up for 5 years.

\section{Setting}

A psychiatric hospital in north London, UK.

\section{Patients}

93 patients aged $16-54$ years (mean age 29 y, $42 \%$ white, $41 \%$ black, $12 \%$ Asian, $5 \%$ other ethnic groups) who made their first contact for psychosis between July 1991 and June 1992. Exclusion criteria were symptoms due to alcohol disorders or other organic syndromes; or had received treatment for symptoms or had contact with any helping agency for symptoms within the previous 3 months.

\section{Assessment of prognostic factors}

At baseline, psychiatric symptoms were assessed using the Present State Examination (PSE), 9th edition and the Syndrome Checklist (SCL). Demographic details were also gathered. Ethnic group was self assigned or assigned by the interviewer.

\section{Main outcome measures}

Psychiatric symptoms (assessed using the PSE and SCL), pattern of illness, demographic details, treatment and compliance, contact with services, admissions to hospital, detentions under the Mental Health Act 1983, and police contact were assessed at 1 and 5 years of follow up.

\section{Main results}

At 5 years, the age adjusted incidence rate ratio of schizophrenia was 4.7 (95\% CI 2.6 to 8.5 ) for black people compared with white people and 4.5 (CI 2.1 to 9.8) for Asians compared with whites. The corresponding incidence rate ratios for non-affective psychosis were 4.3 (CI 2.6 to 7.2) and 3.2 (CI 1.5 to 6.8). A diagnosis was assigned to $91 \%$ at 1 year and $85 \%$ at 5 years. Patients assigned schizophrenia at recruitment were most likely to remain unchanged (at 5 years odds ratio 31, CI 4 to 247). Compared with the diagnosis given at recruitment, 23 patients at 1 year $(27 \%)$ and 27 at 5 years $(35 \%)$ were assigned a different category. Change in diagnostic category was not associated with ethnic group (at 1 year $p=0.1$, at 5 years $p=0.8$ ). The course of illness was rated for $87 \%$ at 1 year and $85 \%$ at 5 years. 20 patients $(24 \%)$ had complete recovery with no further episodes at 1 year, but only 8 sustained this to 5 years. Ethnic group was not associated with a good outcome (at 1 year $\mathrm{p}=0.7$, at 5 years $\mathrm{p}=0.4)$. In the 5 th year when reviewing contact with services, social factors, and treatment, black people were more likely than others to be detained $(\mathrm{p}=0.01)$, brought to hospital by the police $(p=0.005)$, and given emergency injections $(p=0.02)$.

\section{Conclusion}

Ethnicity was not associated with course of the psychotic illness or diagnostic stability over 5 years.
Source of funding: Department of Health, UK

For correspondence: Professor M King, Department of Psychiatry and Behavioural Sciences, Royal Free and University College Medical School, Royal Free Campus, Rowland Hill Street, London NW3 2PF UK. Fax +44(0)1718302468.

\section{COMMENTARY}

Of interest to the clinician is an answer to the following question: do ethnic minority patients such as people of Caribbean origin have a different course of illness than their white British peers? This question clearly has face validity because ethnic minority status is associated with differences in pathway to care, service uptake, risk of misdiagnosis, treatment adherence, and cultural influences on perceptions of illness aetiology and treatment. The combined effect of these differences may well influence course and outcome. How helpful are the studies by Goater $e t$ al and Harrison et al?

Authors of both studies refer to previous publications for a clear description of the ethnic composition of their samples. Goater et al combine a group of patients of Caribbean and African origin on the basis of their appearance into a "black" group, whereas a group of Indian, Pakistani, and other Asians are grouped together on the basis on their place of origin into an "Asian" group. Finally, a group of patients of Greek, Turkish, Irish, British, and other European origin is again grouped on the basis of appearance into a "white" group. Of the white group, actually fewer than half were of British white origin. The question arises of how meaningful is the comparison between the black and white groups in this study. Both groups are defined on the basis of appearance, making the comparison more a racial than an ethnic one. The fact that more than half of the white group were of non-British origin means that differences due to ethnic minority status, if they existed, would be difficult to detect in this small sample. continued on next page 